\title{
The Clinical Expression and Implication of Plasma miRNAs in Patients with Atrial Fibrillation
}

\section{A Expressão e Implicação Clínica de miRNAs Plasmáticos em Pacientes com Fibrilação Atrial}

\author{
Kamilo Mesquita Dantas ${ }^{1,{ }^{*}}$ Heitor Tomé Rezende ${ }^{1}$, Antônio Menezes da Silva Junior ${ }^{1}$, \\ Hermínio Sobrinho ${ }^{1}$, Pedro Paulo Clark de Oliveira ${ }^{1}$
}

ORCID ID

Dantas KM (D) https://orcid.org/0000-0001-5792-5360

Rezende HT (D) https://orcid.org/0000-0003-0988-3703

\author{
Silva Junior AM (D) https://orcid.org/0000-0003-1751-5206 \\ Sobrinho H (D) https://orcid.org/0000-0002-7521-3700 \\ Oliveira PPC (D) https://orcid.org/0000-0001-7625-4515
}

\begin{abstract}
Introduction: circulating miRNA were identified as potential biomarkers in cardiac arrhythmias. It is intended to evaluate, in patients with atrial fibrillation (AF), the expression of miRNAs in plasma, their clinical repercussion and their prognostic factor. Materials and Methods: PubMed, Cochrane and Google Scholar platforms were used as a data source. Eligibility criteria: miRNAs as direct biomarkers in the clinical expression of AF; all languages. Last search: 19/09/2019; studies involving other comorbidities were excluded; pharmacological focus; in animals, in patients already submitted to cardiac procedures and review articles. Plasma collection and storage, RNA isolation, data analysis, real-time PCR, miRNA target prediction and statistical analysis were performed in the studies. Results: The studies generated $n=332$ patients: with AF and control group. In patients with AF there was an increase in the expression of miR-1266, miR-4279, miR-892a, miR-3149, miR-155, miR-146b5p, miR-19b and a reduction in miR-3171, miR-3664-5p and miRNA -150. Discussion: it was noted that the regulation of inflammatory mediators, ion channels and cardiac morphophysiology are related to the expression of miRNA. Conclusion: Therefore, the levels of changes in miRNAs may reflect the severity of the clinical and pathophysiological progression of AF, and can be used as predictors of AF.
\end{abstract}

KEYWORDS: Biomarkers; Atrial Fibrillation; MicroRNAs.

\begin{abstract}
RESUMO
Introdução: miRNA circulantes foram identificados como potenciais biomarcadores em arritmias cardíacas. Assim, pretende-se avaliar, em pacientes com fibrilação atrial (FA), a expressão de miRNAs no plasma, a sua repercussão clínica e seu fator prognóstico. Materiais e Métodos: plataformas PubMed, Cochrane e Google Scholar foram usadas como fonte de dados. Critérios de elegibilidade: miRNAs como biomarcadores diretos na expressão clínica da FA; todos idiomas. Última busca: 19/09/2019; excluídos estudos envolvendo outras comorbidades; foco farmacológico; em animais ;em pacientes já submetidos a procedimentos cardíacos e artigos de revisão. Nos estudos foram realizados a coleta e armazenamento de plasma, isolamento de RNA, análise de dados, PCR em tempo real, previsão de alvo do miRNA e análise estatística. Resultados: Os estudos analisados geraram um $n=332$ pacientes separados em: portadores de FA e grupo controle. Em pacientes com FA houve aumento da expressão do miR-1266, miR-4279, miR-892a, miR-3149, miR-155, miR-146b5p, miR-19b e redução de miR-3171, miR-3664-5p e miRNA-150. Discussão: notou-se que a regulação de mediadores inflamatórios, canais iônicos e morfofisiologia cardíaca estão relacionados com a expressão dos miRNA. Conclusão: logo, os níveis de alterações de miRNAs podem refletir a gravidade do avanço clínico e fisiopatológico da FA, podendo ser utilizados como preditores da FA. .
\end{abstract}

PALAVRAS-CHAVE: Biomarcadores; Fibrilação Atrial; MicroRNAs.

1.Pontifícia Universidade Católica de Goiás - Escola de Ciências Médicas, Farmacêuticas e Biomédicas Goiânia (GO), Brazil.

Received: Nov. 18, 2019 | Accepted: Jan. 27, 2020

*Correspondence author: kamilomesquitadantas@hotmail.com

Section Editor: José Tarciso Medeiros de Vasconcelos 


\section{INTRODUCTION}

Atrial fibrillation (AF) is a supraventricular arrhythmia in which a complete disorganization in the atrial electric activity occurs. It occurs when a diffuse and disorganized pattern of electrical activity in the atria suppresses or replaces the normal sinus mechanism. This disorder is one of the main causes of morbidity, mortality and public health spending ${ }^{1}$.

In the last two decades, $\mathrm{AF}$ has become a major public health problem at great cost to health resources. It has an important impact on life quality, especially due to its clinical consequences, thromboembolic phenomena and cognitive alterations ${ }^{1}$. In this regard, studies involving microRNAs (miRNAs) appear as a new perspective in early AF diagnosis.

Atrial fibrillation is the most frequent cardiac arrhythmia in clinical practice and its prevalence in the general population was estimated between 0.5 and $1 \%$. In the United States, 2.3 million people have fibrillation, and that number is expected to rise to 5.6 million by 2050. It is also associated with a five-fold increased risk of stroke and is estimated to cause $15 \%$ of all strokes ${ }^{1}$.

Genetic factors participate in the generation of electrophysiological factors and substrates that determine individual susceptibility to the onset and progression of $\mathrm{AF}^{2}$. The presence of single nucleotide polymorphisms (SNPs) can alter the expression of microRNAs and proteins and is established as a contributing factor in complex heart diseases ${ }^{3}$.

In recent years, analysis of candidate genes and the complete genome in AF patients has been conducted in order to understand the molecular mechanisms underlying the development of this disease ${ }^{1}$. Several studies have demonstrated the role of post-transcriteria regulatory mechanisms, including microRNAs in the etiology of arrhythmogenesis ${ }^{2}$. Recent researches show that miRNAs regulate the excitability and several physiological traits of cardiac cells, including automaticity, $\mathrm{Ca}^{2+}$ flow, conduction and repolarization, and are strongly linked to electrical and structural remodeling, which are the main factors in the promotion and progression of arrhythmias ${ }^{2}$. In addition, new studies have demonstrated the relationship between miRNAs and the development of inflammatory and fibrotic processes, which are highly correlated to AF. This led to a paradigm shift from an "electrical" problem to something more "structural"4. Thus, miRNAs are understood as potential biomarkers for the development and maintenance of this arrhythmia.

\section{OBJECTIVES}

The objective of this study was to evaluate the expression of miRNAs in plasma, their clinical repercussion and their prognostic factor in AF patients. This review contributes to the understanding of the process that triggers AF by encouraging new studies to evaluate the application of these molecules as biomarkers of this disease.

\section{METHODOLOGY}

This is a systematic review according to PRISMA recommendation in order to analyze studies on the expression of miRNAs in plasma, their clinical repercussion and their prognostic factor in AF patients. There was no language selection or time delimitation, and the last search was made on September 19,2019. Studies involving other comorbidities, with a pharmacological focus, in animals, in patients already submitted to heart procedures, and review articles were excluded. Thus, studies that brought miRNAs as biomarkers in the clinical expression of AF were prioritized, allowing the analysis of the direct correlation between these molecules and the disease.

The search for articles occurred until September 19, 2019 using the keywords "miRNAs", "atrial fibrillation" and "biomarkers" (always in additive character), in the platforms: PubMed and Cochrane, where 55 and 100 articles were screened respectively. At PubMed, it was typed in the search bar: miRNAs AND atrial fibrillation AND biomarkers. There was no language selection, specific period or specification of publication type. The search was made until September 19, 2019, and it generated 55 articles. Google Scholar was also used as a search platform, however, by following the same strategy of the other platforms, the number of articles exceeded 1,600 , interfering in the quality of this review. Thus, a periodization was stipulated (selecting articles from the year 2015 until the date of the last search of the other 
platforms) using the keywords "miRNAs expression", "atrial fibrillation" and "biomarkers", which resulted in 112 articles, totaling an $n=267$ articles screened for this review.

Among the 267 articles screened, the duplicates were removed, leaving 243 articles that were independently read by two of the authors of this review, under the guidance of a third author. At this stage, the studies were selected from the reading of the title and abstract of each one, applying the eligibility criteria and eliminating articles that analyzed other comorbidities concomitant to AF; studies with focus on other diseases such as thyroidopathies, diabetes mellitus, neoplasms, depression, atherosclerosis and coagulopathies; and articles whose focus were pharmacological studies, leaving 27 articles, which were read in full independently by the authors. There were new exclusions: research articles developed on animals, articles with preliminary results, systematic review articles, texts published as abstracts or editorials. Studies on specific populations were also excluded (AF in marathoners; AF in patients undergoing ablation, revascularization or any other cardiovascular procedure; $\mathrm{AF}$ in individuals using anticoagulants; $\mathrm{AF}$ in patients who suffered stroke). In addition, articles addressing miRNAs as atherosclerotic biomarkers, strokes and thrombotic events were removed from this review. Consequently, three remaining articles met the eligibility criteria of this review, bringing a direct relation of miRNAs as biomarkers in the clinical expression of AF.

A comparative chart was established between the three studies showing author, year of publication, type of study, participant population, applied methodology and results. It was possible to highlight the difference in the expression of miRNAs in AF and control group patients, differences in the expression of miRNAs according to the type of AF and AF-related genes (related to inflammatory response, influence on calcium ion channels, involved in apoptosis and fibrosis of the heart tissue).

According to Risk of Bias in Systematic Reviews (ROBIS), the review is evaluated as low bias potential.

\section{RESULTS}

The study of Xu et al..$^{5}$ evaluated a certain population in the Hospital Shijitan of Beijing, from January 2011 to June 2015. Ninety patients with a median age of $72.17 \pm 4.76$ years and AF in preparation for radiofrequency ablation were classified as groups of paroxysmal, persistent or permanent AF. The mean age of the 90 individuals in the control group was $69.40 \pm 5.86$ years. Each patient had more than five electrocardiograms at different times, supporting his diagnosis. It was found in the study that the differential expression of miRNAs in coronary circulation in patients with AF of three types: the miRNAs expression in coronary sinus blood (CSB) may better reflect the actual metabolism, expression and regulatory status of miRNAs in AF patients; levels of change in miRNA may reflect the severity of clinical and pathophysiological progress of AF; and that miR-3171, miR-892a, and miR-3149, expressed variably in the early and late stages of the disease, may be used as biomarkers for early diagnosis of $\mathrm{AF}$.

A study of $\mathrm{Xu}$ et al. ${ }^{5}$ compared the peripheral blood of normal individuals (PB group). MiR-3171 levels were lower in AF patients, indicating that it is secreted mainly by tissues other than the myocardium, and may participate in the regulation of the disease through coronary circulation. The continuous decrease of miR-3171 in patients with persistent and permanent paroxysmal AF makes it possible for it to become an efficient biomarker. Additionally, the expression of miR-892a and miR-3149 was greater in the PB group than in patients than in the CSB group, as well as in the normal control group (HC). There was no difference between the CSB and the $\mathrm{HC}$ groups. This indicates that they were produced by tissues other than heart cells and act as regulators in the coronary circulation. Although both have been reduced in the patients' $\mathrm{CSB}$, but increased in $\mathrm{PB}$, they will probably become diagnostic markers of AF. In addition, it is also necessary to confirm that many factors such as aging, hypertension, dyslipidemia and other systemic diseases, states of sympathetic activation and inflammation can affect the outcome.

The study conducted by Liu et al. ${ }^{6}$ evaluated patients between March and December 2011. A total of 105 participants (61 men, 44 women) with a median age of 51 were registered. All AF patients were monitored by electrocardiography for 7 days to support the diagnosis. It was found that the plasma level of miRNA-150 in AF patients was substantially lower than in healthy people. As a result, the reduced miRNA-150 in circulation 
was significantly associated with AF. As a next step, the study sought to determine whether miRNA-150 was linked to AF pathogenesis. The study sought to identify correlations in clinical samples between miR-150 expression levels and important protein-coding genes involved in AF pathogenesis. A target search for miR-150 was performed using four databases (TargetScan, miRanda, Starbase Clip-seq and miRDB). It was found that at least 18 genes were related to AF. Among them, 11 genes were related to the inflammatory response system, 4 played a role in calcium ion channels, 2 were involved in apoptosis and 1 related to fibrosis of the heart tissue.

The inflammation was not only a marker of the incident AF, but is also mechanically involved in inducing the fibrillation process. The predicted target genes (IL-6, IL-18, TNF- $\alpha$ and TGF- $\beta$ ) increased significantly in AF patients. Cytokines such as these are produced by activated cells, usually monocytes and macrophages that also secrete miRNA-150 in response to inflammatory stimuli, with the objective of reducing inflammation. They are fundamental in the activation of the inflammatory cascade and in the production of acute phase proteins. One such acute phase protein that is the focus of much research is the $\mathrm{C}$-reactive protein (CRP). These findings suggest a negative correlation between the expression of inflammatory cytokines and miR-150. Thus, the study found that plasma levels of $\mathrm{CRP}$ in patients with persistent $\mathrm{AF}$ were higher than in patients with paroxysmal $\mathrm{AF}$, and the levels in both groups of AF were higher than in the control group. MiRNA-150 had a high negative correlation with the inflammatory response and, consequently, with $\mathrm{CRP}^{6}$.

Liu et al. ${ }^{6}$ demonstrated a specific set of miRNAs in AF patients with single expression. Expression levels of miRNA-146a, miR-150, miRNA-375, and miRNA-19a were significantly reduced in AF patients. However, only the expression of miRNA-150 was important enough to deserve further evaluation. Significantly lower expression levels of miRNA-150 were found in the population evaluated with $\mathrm{AF}$, which was identified as a predictor of the disease (OR 1.96, 95\% CI 1.5 to $3.57, P<0.001)$. The study concluded that, due to the limited number of samples used in the analysis, its ability to confirm the diagnostic power of microRNA and its value for clinical testing in patients with $\mathrm{AF}$ was impaired. Future prospective studies in larger cohorts are needed to establish the roles of these miRNAs in atrial fibrillation ${ }^{6}$.

A total of 47 individuals were evaluated by Wang et al. ${ }^{7}$ Expression profiles of miRNAs in the left atrial appendix (LAA) were obtained in 30 patients with paroxysmal AF. The mean age was $48.5 \pm 7.2$ years, and $63 \%$ were male. The period since the first diagnosis of $\mathrm{AF}$ was \pm 2 years.

In particular, miR-155, miR-146b-5p and miR$19 \mathrm{~b}$ demonstrated the most pronounced changes. These results indicated the possible roles of miRNAs in $\mathrm{AF}$ progression. It was found that miR-155, miR-146b-5p and $\mathrm{miR}-19 \mathrm{~b}$ are considerably elevated in patients' LAA with AF compared to the control group. A group of miRNAs regulates genes that encode cardiac ion channel proteins and other relevant genes, and several studies have reported the direct involvement of miRNAs in controlling cardiac excitability and arrhythmogenesis. Some of these miRNAs have been shown to be directly involved in $\mathrm{AF}$ and some are considered to have a potential role in the pathogenesis of fibrillation according to their target genes ${ }^{7}$.

To determine factors that influence the levels of miRNAs, Wang et al. ${ }^{7}$ analyzed the association between miRNAs and the basal characteristics of patients. There were significant positive correlations between the expression of miR-146b-5p and miR-155 and the mean left atrial diameter (LAD) of patients with $\mathrm{AF}$, in addition to CRP plasma levels and $\mathrm{AF}$ duration, respectively. Interestingly, LAA levels, AF duration, and CRP levels are considered to be predictors of increased risk for $\mathrm{AF}$ recurrence.

During the study, miR-155, miR-146b-5p and miR-19b were significantly increased in patients with non-valvar PAF. More importantly, significantly higher levels of expression of miR-155 and miR-146b-5p were also found, independently associated with LAD, AF duration, and CRP levels. However, only the expression level of miR-155 and miR-146b, the LA size, and the AF duration were able to significantly predict recurrence of AF in multivariate analysis ${ }^{7}$.

Curiously, miR-155, which is reported to be involved in cardiovascular diseases, showed the greatest increases in AF patients compared to the control group. The miR-155 can directly influence arrhythmia results by directing 
the critical ion channel gene expression, including CACNA1C and KCNA4, which encode the $₫ 1 \mathrm{c}$ L-type $\mathrm{Ca}^{2+}$ channel $\left(\mathrm{I}_{\mathrm{CaL}}\right)$ and the transient external initial component of the potassium current $\left(\mathrm{I}_{\mathrm{to1}}\right)$, respectively. The authors found that the expression of CACNA1C was significantly lower in the AF group than in the control group, not only for the protein level, but also for the mRNA level. Also, positive regulation of the miR-155 can play a role in inflammation. Although the mechanism regulating the function and expression of miRNA was not fully understood during the analysis, currently available information (i.e., superexpression in AF patients) has allowed the recognition of miR-155 as a gene of potentially paramount clinical importance in the diagnosis and treatment of AF. Thus, the evaluation of mRNA-155 in tissues or biological fluids can be used as a biochemical parameter for detection and prognosis of $\mathrm{AF}^{7}$.

It is also believed that miRNA-146 is a mediator of inflammation along with miRNA-155. Wang et al. ${ }^{7}$ evaluated that the expression of $\mathrm{miR}-146$ is positively regulated by inflammatory factors such as interleukin-1 and tumor necrosis factor. The miR-146 regulates a series of targets, which are mainly involved in Toll-like receptors pathways, which cause a cytokine response as part of the innate immune system. Recently, it was reported that the miR-146b-5p was positively regulated in AF patients, which is consistent with the study findings. Potential targets for miR-146b-5p include TGIF1, MMP16 and TIMP4, which are reported as participants in the formation of fibrosis in cardiomyocytes, and these can be expected to contribute to AF.

The MiR-19b was also evaluated, and it was found that it also participates in inflammatory responses, improving or repressing the expression of pro-inflammatory mediators. The miR-19b is an important protagonist in this phenomenon, positively regulating the nuclear factor kappa-B (NF-kB) activity. Depletion of miRNA inhibits cytokine production from NF-kB. In addition, it is directly involved in modulating the expression of various negative $\mathrm{NC}-\mathrm{kB}$ signal regulators, indicating their importance. This miRNA was not extensively studied, but the data from the study suggest a possible role in AF pathology, which is yet to be identified with more emphasis ${ }^{7}$.

\section{DISCUSSION}

Liu et al. ${ }^{6}$ identified a relationship in clinical samples between miR-150 expression levels and important protein-coding genes involved in the inflammatory response associated with $\mathrm{AF}$ pathogenesis. It was observed that at least 11 genes were related to the inflammatory response system. Moreover, inflammation was not only a marker of incident AF, but is also mechanically involved in the propensity to this disease. The predicted target genes (IL-6, IL-18, TNF- $\alpha$ and TGF- $\beta$ ) increased significantly in AF patients. Cytokines such as these are produced by activated cells, usually monocytes and macrophages, which also secrete miRNA-150 in response to inflammatory stimuli ${ }^{6}$. These findings suggest a negative correlation between the expression of inflammatory cytokines and miR-150.

Wang et al. ${ }^{7}$ also evaluated inflammatory factors and found that MiR-146 is an inflammation mediator together with MiR-155. The study evaluated that the expression of miR-146 is positively regulated by factors such as interleukin-1 and tumor necrosis factor. In addition, the potential targets of miR-146 are participants in the formation of fibrosis in cardiomyocytes and they can be expected to contribute to AF. The MiR-19b was also evaluated in the study, and it was found that it also participates in inflammatory responses, improving or repressing the expression of pro-inflammatory mediators. The miR-19b participates in the regulation of nuclear factor kappa-B (NF-kB) activity, which is highly related to inflammatory processes in cardiomyocytes and, consequently, to AF.

Circulating miRNAs in $\mathrm{AF}$ patients were sequenced by Liu et al. ${ }^{6}$ and the expression levels of miR-146a, miR-150 were remarkably reduced. The authors concluded that a reduction in circulating miR-150 was significantly associated with AF. In addition, they found that plasma CRP levels were negatively correlated to plasma levels of miR-150, which was also evidenced by Xu et al. ${ }^{5}$.

According to Korantzopoulos et al. ${ }^{8}$, one of the main factors and conditions of cardiovascular risk associated with $\mathrm{AF}$ is inflammation. Inflammation can lead to AF, but $\mathrm{AF}$ also promotes inflammation, leading to a vicious and progressive cycle. To support the process of chronic inflammation in cardiac arrhythmia, several studies have 
Table 1. Description of the results.

Xu et al. (2016)
Different expression of miRNAs in AF patients and control group:

- miR-1266 and miR-4279, increased 11.38 and 2.38 times, respectively. In contrast, miR-3171 and miR-3664-5p decreased 0.09 and 0.14 times, respectively.

- miR-892a and miR-3149 increased 2.32 and 2.35 times, respectively, while miR-3171 decreased 0.21 times
Differences in miRNAs expression in patient groups

ParoAF, PersAF and PermAF:

- miR-1266 increased 5.55-, 8.41- and 4.70 times, and miR-3171 decreased 0.43-, 0.47- and 0.32 times in ParoAF, PersAF and PermAF groups, respectively.

- ParoAF compared to the PersAF group did not show significance, but there was one difference.

Liu et al. $(2012)^{6}$

- It was found that the plasma level of miRNA-150 in AF patients was substantially lower than in healthy people.

- 18 genes were related to AF. Among them, 11 genes were related to the inflammatory response system, 4 played a role in the calcium ion channels, 2 were involved in apoptosis and 1 was related to fibrosis of the heart tissue.

- IL-6, IL-18, TNF- $\alpha$, and TGF- $\beta$ increased significantly in AF patients (miRNA-150 aims to reduce the release of these cytokines).

- In the population evaluated with AF, significantly lower levels of expression of miRNA-150 were found, and thus it was identified as a predictor of the disease.

- miR-155, miR-146b-5p and miR-19b are considerably high in AF patients compared to the control group.

- MiR-155 can directly influence arrhythmia results by directing the gene expression of the critical ion channel, including CACNA1C and KCNA4, which encode the Ca2+ L-type cardiac channel.

Wang et al. (2015)

- miRNA-146 is an inflammation mediator along with miRNA-155. MiR-146 expression is positively regulated by inflammatory factors such as interleukin-1 and tumor necrosis factor.

- MiR-19b was also evaluated and it was found that it also participates in inflammatory responses, improving or repressing the expression of pro-inflammatory mediators.

indicated the association between autoimmune disorders correlated to cardiac problems. A crescent number of studies indicate that inflammatory mediators promote remodeling of the atrial structure and electrical changes. The mechanisms involved are: atrial fibrosis, modulation of the gap junction and intracellular abnormalities in calcium handling.

Guo et al. ${ }^{9}$ brought several prospective epidemiological studies that confirmed that inflammation may confer an increased risk of AF. Among them, a large cohort involving 25,883 participants from Women's Health Study that showed inflammatory biomarkers (CRP, soluble intercellular adhesion molecule- 1 and fibrinogen) associated with increased incidence of AF in middle-aged women. These abnormalities increase atrial ectopic activity and decrease atrial conduction, impairing normal atrial impulse propagation and promoting reentry. Such facts were proven by Liu et $a 1 .{ }^{6}$, who related the suppression of miRNA-150, which is related to the blockade of inflammation and patients who present AF.

Wang et al. ${ }^{7}$ demonstrated that miR-155, miR-146b-5p and $\mathrm{miR}-19 \mathrm{~b}$ are considerably elevated in AF patients compared to the control group. A group of miRNAs regulates genes that encode cardiac ion channel proteins and, therefore, these miRNAs have shown to be directly involved in the pathogenesis of AF. The research found that the miR-155 was increased in AF patients compared to the control group. The miR-155 can directly influence 
Table 2. Results.

\begin{tabular}{|c|c|c|c|c|c|}
\hline Author & Year & Type of Study & Number of patients & Statistical analysis & Methods \\
\hline Xu et al. ${ }^{5}$ & 2016 & Control case & $\begin{array}{l}180 \text { patients ( } 90 \text { with } \\
\text { AF and } 90 \text { as control } \\
\text { group). }\end{array}$ & $\begin{array}{l}\text { The significance threshold value } \\
\text { used to define the positive or } \\
\text { negative regulation of miRNAs was } \\
\text { a variation }>1.5 \text {, with a value of } P \\
<0.05 \text { calculated by the } t \text {-test and } \\
\text { analysis of variance. }\end{array}$ & $\begin{array}{l}\text { Performing plasma collection } \\
\text { and storage, RNA isolation, } \\
\text { MicroRNA matrix and data } \\
\text { analysis, real-time CRP. } \\
\text { miRNA target prediction and } \\
\text { statistical analysis. }\end{array}$ \\
\hline Liu et al. ${ }^{6}$ & 2012 & Cohort study & $\begin{array}{l}105 \text { participants (10 } \\
\text { with AF, } 5 \text { from the } \\
\text { control group and } 90 \\
\text { random patients as } \\
\text { test using qRT - CRP). }\end{array}$ & $\begin{array}{l}\text { miRNA-150 expression: (odds ratio } \\
\text { [OR] 1.96, 95\% confidence interval } \\
\text { [CI] 1.5 to 3.57, P < 0.001), age (OR: } \\
\text { 1.1, Cl: } 95 \% 1.36 \text { to } 2.73), P<0.001 \text { ) } \\
\text { and left atrium diameter (LAD) (OR: } \\
\text { 1.5, Cl: } 95 \% 1.36 \text { to 1.8, P < 0.001). } \\
\text { Plasma CRP levels were negatively } \\
\text { correlated to miRNA-150 plasma } \\
\text { levels: } 95 \% \text { confidence interval [Cl] } \\
\text { 1.5 to 3.57, P < 0.001), age (OR: 1.1, } \\
\text { Cl: } 95 \% 1.36 \text { to } 2.73, P<0.001) \text { and } \\
\text { left atrium diameter (LAD) (OR: } 1.5 \text {, } \\
\text { Cl: } 95 \% 1.36 \text { to 1.8, P < 0.001). }\end{array}$ & $\begin{array}{l}\text { Performing plasma collection } \\
\text { and storage, RNA preparation, } \\
\text { deep sequencing and qRT- } \\
\text { CRP, MiRNA target prediction, } \\
\text { measurement of hs-CRP and } \\
\text { statistical analysis. }\end{array}$ \\
\hline Wang et al. ${ }^{7}$ & 2015 & Control case & $\begin{array}{l}\text { A total of } 47 \text { individuals } \\
\text { ( } 30 \text { AF patients and } 17 \\
\text { as a control group). }\end{array}$ & $\begin{array}{l}\text { miRNA-155 [Hazard Ratio }[H R], \\
\text { 1.113; } P=0.037) \text {. } \\
\text { miR-146b-5P (HR, 1.646; } P=0.030) \\
\text { expression levels, LAD* (HR, 1.036; } \\
P=0.039) \text { and duration of AF (HR, } \\
1.216 ; P=0.044) \text { were found to } \\
\text { significantly predict recurrence of AF. } \\
\text { *LAD: left atrium diameter }\end{array}$ & $\begin{array}{l}\text { Performing total isolation } \\
\text { of RNA (including miRNA), } \\
\text { reverse MiRNA transcription } \\
\text { and quantitative CRP, } \\
\text { statistical analysis of miRNA } \\
\text { data, quantitative analysis of } \\
\text { real-time CRP, Western Blot } \\
\text { and statistical analysis. }\end{array}$ \\
\hline
\end{tabular}

arrhythmia by directing the gene expression of the critical ion channel, including CACNA1C and KCNA4 (genes responsible for the formation of calcium channels), which encode the cardiac L-type calcium channels and the initial component of the transient external potassium current. The authors evaluated in the study that the expression of CACNA1C was significantly lower in the AF group than in the control group.

Experimental mapping studies and studies in humans have demonstrated that persistent $\mathrm{AF}$ is usually related to excitation waves that propagate through the atrium. Perpetuation of AF is facilitated by the existence or abnormal development of atrial tissue capable of maintaining arrhythmia, with the number of waves that can determine the stability of AF. Reentry into the atrial myocardium is facilitated by the slowing of conduction and shortening of the refractory period ${ }^{8}$. Both were demonstrated in $\mathrm{AF}$ patients, further contributing to arrhythmogenesis. Shortening of the atrial action potential, reduced expression of L-type calcium channels and atrial fibrosis were also demonstrated, facts proven by Wang et al. ${ }^{7}$, who evaluated the participation of miRNAs in the ionic channels functioning of cardiac tissue.

Xu et al. ${ }^{5}$ compared the blood collected in the coronary sinus of AF patients with the peripheral blood of normal individuals. The miR-3171 levels were lower in AF patients, indicating that it is secreted mainly by other tissues besides myocardium and may participate in the regulation of the disease through coronary circulation. Therefore, it is also necessary to confirm that many factors such as aging, hypertension, dyslipidemia and other systemic diseases, states of sympathetic activation and inflammation can affect the results. This fact has already been 
proven in other studies, such as that of Korantzopoulos et al. ${ }^{8}$, in which miRNA are highly related to the stress of the body, participating in the regulation of inflammatory processes. Thus, new studies evaluating the role of miRNA in processes that promote aggressions to the organism should be conducted in order to evaluate their correlations not only with AF, but with several pathologies, as shown in Fig. 1.

\section{CONCLUSION}

In short, it is understood that miRNAs are in fact positively or negatively correlated to atrial fibrillation, acting directly on morphology and/or cardiac function. Thus, the union of these studies reinforces the idea of the importance of these molecules as biomarkers of the disease, giving new directions to research on the diagnosis, development, maintenance and prognosis of atrial fibrillation.

However, the ability of this review to confirm the diagnostic power of miRNAs is still limited, given the small number of individuals in the reviewed studies. Another important point that needs to be addressed is the pathophysiological mechanisms to which these molecules are involved, allowing a more punctual and effective diagnostic approach.

Estratégia de busca:

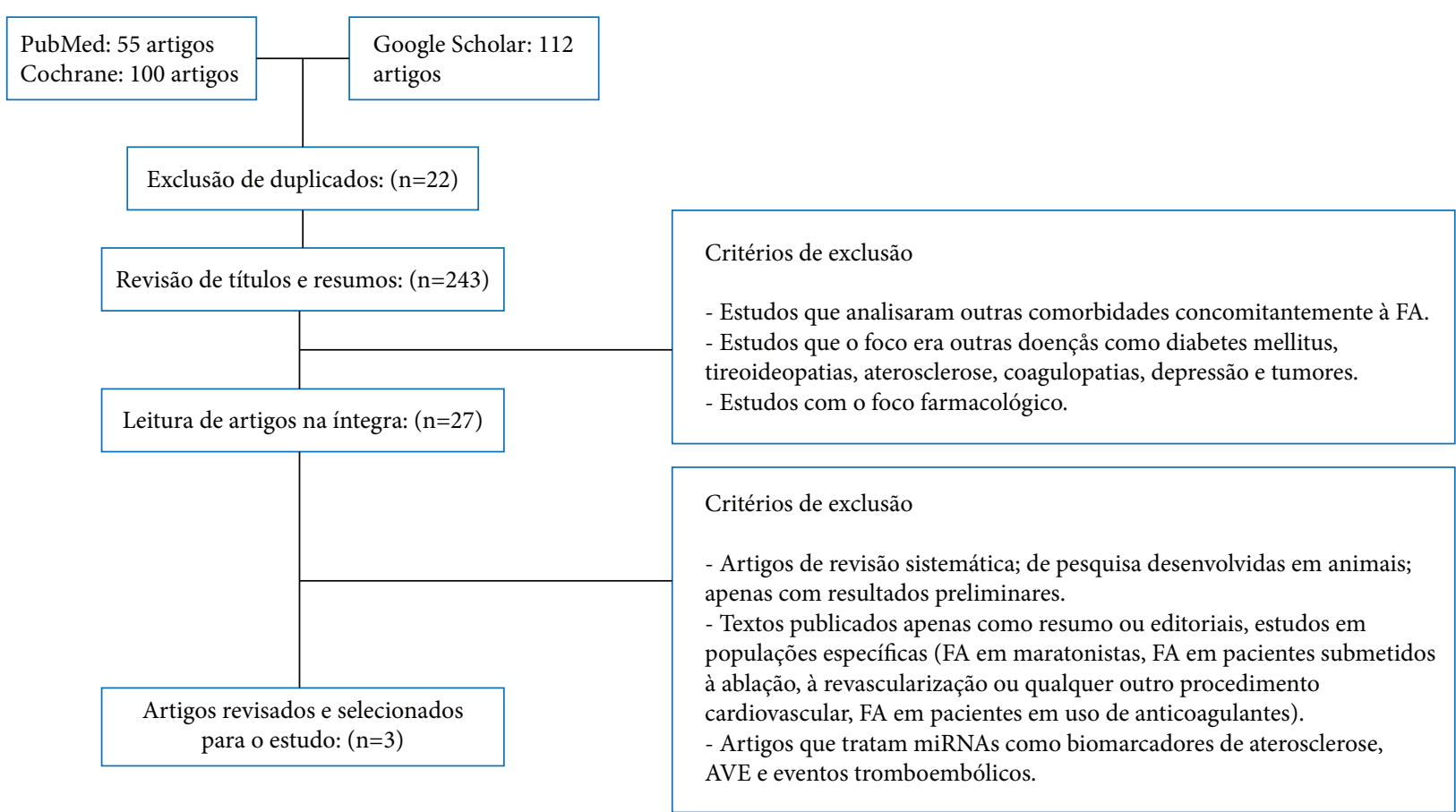

Figure 1. PRISMA flow diagram, exclusion criteria.

\section{REFERENCES}

1. Carlos, A., Pimenta, E., \& Drager, L. F. (2016). II diretriz de Fibrilação Atrial, 106, 44-53.

2. Shi KH, Tao H, Yang J-J, Wu J-X, Xu S-S, Zhan H-Y. Role of microRNAs in atrial fibrillation: New insights and perspectives. Cell Signal, 2013;25(11):2079-84. https://doi. org/10.1016/j.cellsig.2013.06.009

3. Cooley N, Cowley MJ, Lin RCY, Marasco S, Wong C, Kaye DM et al. Influence of atrial fibrillation on microRNA expression profiles in left and right atria from patients with valvular heart disease. Physiol Genomics, 2012;44(3):211-9. https:// doi.org/10.1152/physiolgenomics.00111.2011

4. Boos CJ, Anderson RA, Lip GYH, Is atrial fibrillation an inflammatory disorder? Eur Heart J, 2006;27(2):136-49, https://doi.org/10.1093/eurheartj/ehi645

5. Xu G, Cui Y, Jia Z, Yue Y, Yang S. The values of coronary circulating miRNAs in patients with atrial fibrillation. PLoS ONE, 11(11):e0166235. https://doi.org/10.1371/journal. pone. 0166235 
6. Liu Z, Zhou C, Liu Y, Wang S, Ye P, Miao X, Xia J. The Expression Levels of Plasma micoRNAs in Atrial Fibrillation Patients. PLoS ONE, 2012;7(9): e44906. https://doi. org/10.1371/journal.pone.00449067.

7. Wang J, Song S, Xie C, Han J, Li Y, Shi J, et al. MicroRNA profiling in the left atrium in patients with non-valvular paroxysmal atrial fibrillation. BMC Cardiovasc Disord, 15(97):1-7. https://doi.org/10.1186/s12872-015-0085-2

8. Korantzopoulos P, Letsas KP, Tse G, Fragakis N, Goudis CA, Liu T. Inflammation and atrial fibrillation: A comprehensive review. J Arrhythmia, 2018;34(4):394-401. https://doi. org/10.1002/joa3.12077
9. Guo Y, Lip GY, Apostolakis S. Inflammation in atrial fibrillation. J Am Coll Cardiol, 2012;60(22):2263-70. https:// doi.org/10.1016/j.jacc.2012.04.063

10. Silva, Ananília Medeiros Gomes da. miRNAs circulantes como biomarcadores da fibrilação atrial aguda/Ananília Medeiros Gomes da Silva. - Natal, 2015.

11. Harada $\mathrm{M}$ et al. Role of Inflammation in Atrial Fibrillation Pathophysiology and Management Circ J. ;79(3):495-502. DOI: 10.1253/circj.CJ-15-0138

12. Markides, V., \& Schilling, R. J. (2003). Atrial fibrillation: classification, pathophysiology, mechanisms and drug treatment * 939. 